PAR-LPTHE 01-06

RUNHETC-2000-54

hep-th/0102075

\title{
Supersymmetry with a Ghost Time
}

\author{
Laurent Baulieu ${ }^{\dagger}$ and Marc Bellon \\ Laboratoire de Physique Théorique et Hautes Energies, \\ Universités Pierre et Marie Curie, Paris VI \\ et Denis Diderot, Paris VII \\ and \\ $\dagger$ Dept. of Physics, Rutgers University, New Brunswick, \\ NJ 60637, USA
}

\begin{abstract}
The progress brought to the study of chiral fermions and gauge theories by quantization methods with a bulk time suggests their usefulness in supersymmetric theories. Using superspace methods, we show how an explicitly supersymmetric version of such quantization methods may be given.
\end{abstract}

Postal address: Laboratoire de Physique Théorique et des Hautes Energies, Unité Mixte de Recherche CNRS 7589,

Université Pierre et Marie Curie, boîte postale 126.

4, place Jussieu, F-75252 PARIS Cedex 05 


\section{Introduction}

Since the introduction of stochastic quantization for gauge theories in [1], its interest has been vindicated in a number of publications (see [2] for a review). In recent works [3, 4. 5], the basic ideas of stochastic quantization have been elaborated in a systematic approach, called bulk quantization. It gives a central role to the introduction of a symmetry of topological character. The inclusion of fermionic fields becomes possible. Perturbatively, bulk quantization and the usual quantization methods are equivalent because the observables satisfy the same Schwinger-Dyson equations in both approaches [5]. The interest of bulk quantization is certainly at the non perturbative level.

These recent developments make the application of bulk quantization to supersymmetric field theories promising. In this letter, we present the simplest $N=1$ theory in four dimensions, the Wess-Zumino model of interactions of chiral supermultiplets.

Using previous works [5], we define an action on a space with an additional non compact dimension $t$ that describes the bulk time. The correlation functions for equal $t$ define the correlations in the physical theory. The additional dimension does not take part in the Poincaré group of symmetries. Spinor fields and the algebra of supersymmetry are thus the usual four-dimensional ones. It follows that one can use the superfield formalism to efficiently describe the different supermultiplets and obtain an invariant action. The supersymmetry is linearly realized and the algebra closes offshell, so that we do not have to worry on the preservation of the supersymmetry by dynamical processes. As a side effect, this formalism puts the auxiliary field of the supermultiplet on the same level as the other components, with its own dynamic.

The nice point in this construction is that the requirement of supersymmetry, obtained through the superspace formalism, naturally introduces the kernels acting on the equations of motion of the four-dimensional theory, guaranteeing that each component has the proper behavior. As it is the case in any field theory with fermions, the difficulty of giving to all equations a stochastic interpretation justifies to directly postulate the topological field theory construction of the bulk theory.

We conclude this note by a short account of the extension to the case of gauge invariant models: the gauge symmetry must be enlarged in a superspace formalism and we point to the difficulties in which would run too naive an approach. A full account of supersymmetric gauge theories will be given in a forthcoming publication [6].

\section{Wess-Zumino model}

In this model, the fields are grouped in supermultiplets which are easily described by chiral superfields. In view of our general principles, these fields will depend on the space coordinates $x$, the anticommuting superspace coordinates $\theta, \bar{\theta}$ and the ghost time $t$.

$$
\Phi(t, x, \theta, \bar{\theta}), \quad \bar{D}_{\dot{\alpha}} \Phi=0 .
$$

The model is then characterized by the superpotential $V(\Phi)$, which is at most trilinear for renormalizable interactions. The equation of motions for $\Phi$ and its antichiral conjugate $\bar{\Phi}$ are expressed in superfields as:

$$
0=\bar{D}^{2} \bar{\Phi}+V^{\prime}(\Phi)=\bar{D}^{2} \bar{\Phi}+m \Phi+\frac{1}{2} g \Phi^{2},
$$




$$
0=D^{2} \Phi+V^{\prime}(\bar{\Phi})
$$

Formally, these equations are very similar to the Dirac equations, with $D^{2}$ and $\bar{D}^{2}$ playing the role of the chiral components of the Dirac operator.

According to the principles of bulk quantization, we introduce three fields to complete the quartet $\Phi, \Psi, \chi, B$. A nilpotent BRST operator is defined by $s \Phi=\Psi$, $s \Psi=0, s \chi=B$ and $s B=0 . \Phi$ and $\Psi$ have the same canonical dimension $1 . \chi$ and $B$ have dimension 2. This can be deduced from the fact that $B$ will appear in combination with the equations of motion for $\Phi$ in a field

$$
H=B+\bar{D}^{2} \bar{\Phi}+V^{\prime}(\Phi)
$$

With the additional knowledge that the ghost time $t$ has dimension -2 and that chiral superfields must be integrated on $d^{2} \theta$, which adds one to the canonical dimension and general superfields must be integrated on $d^{4} \theta$, which adds two, we define the following $s$-invariant and supersymmetric action of ghost number zero with only positive dimension coupling constants:

$$
\begin{aligned}
& \int d t d^{4} x d^{2} \theta s\left(\chi \partial_{t} \Phi\right)+\int d t d^{4} x d^{2} \bar{\theta} s\left(\bar{\chi} \partial_{t} \bar{\Phi}\right) \\
& +\int d t d^{4} x d^{4} \theta s(\bar{\chi} H+\chi \bar{H}) \\
& +\int d t d^{4} x d^{2} \theta M s(\chi H)+\int d t d^{4} x d^{2} \bar{\theta} M s(\bar{\chi} \bar{H}) .
\end{aligned}
$$

$M$ is an additional mass parameter introduced by bulk quantization. Physical quantities will not depend on $M$, because the $M$-dependence will appear through a kernel.

We will shortly justify that the field $\phi$ only appears through $\partial_{t} \phi$ and $H$ in eq. ( $($ ) by the invariance under bulk time reversal. The ghost independent part of this action is:

$$
\begin{aligned}
& \int d t d^{4} x d^{2} \theta B \partial_{t} \Phi+\int d t d^{4} x d^{2} \bar{\theta} \bar{B} \partial_{t} \bar{\Phi} \\
& +\int d t d^{4} x d^{4} \theta\left(2 B \bar{B}+B\left(\bar{D}^{2} \bar{\Phi}+V^{\prime}(\Phi)\right)+\bar{B}\left(D^{2} \Phi+V^{\prime}(\bar{\Phi})\right)\right) \\
& +\int d t d^{4} x d^{2} \theta M\left(B^{2}+B\left(\bar{D}^{2} \bar{\Phi}+V^{\prime}(\Phi)\right)\right) \\
& +\int d t d^{4} x d^{2} \bar{\theta} M\left(\bar{B}^{2}+\bar{B}\left(D^{2} \Phi+V^{\prime}(\bar{\Phi})\right)\right)
\end{aligned}
$$

Variation with respect to the superfields $B$ and $\bar{B}$ gives the following matrix equation:

$$
\left(\begin{array}{c}
\partial_{t} \Phi \\
\partial_{t} \bar{\Phi}
\end{array}\right)=\left(\begin{array}{cc}
M & \bar{D}^{2} \\
D^{2} & M
\end{array}\right)\left(\begin{array}{l}
\bar{D}^{2} \bar{\Phi}+V^{\prime}(\Phi)+2 B \\
D^{2} \Phi+V^{\prime}(\bar{\Phi})+2 \bar{B}
\end{array}\right)
$$

These equations look like stochastic equations for the superfields $\Phi$ and $\bar{\Phi}$ with driving noises $B$ and $\bar{B}$, but this interpretation (that would hold true for a genuine scalar field theory) does not stand out. In particular, $B$ mixes with the physical field $\Phi$. The action (5) is quadratic in the field $B$, but as in the fermionic case, the quadratic term 
$\int B \bar{B}$ contains space derivatives. The propagator is a matrix with $\Phi-\Phi$ and $B-\Phi$ propagations.

From the superfield $\Phi$, three fields can be defined by taking the $\theta=\bar{\theta}=0$ value (denoted by the symbol $\mid$ ) of the fermionic derivatives of $\Phi$ :

$$
\begin{aligned}
A(x, t) & =\Phi(x, t, \theta, \bar{\theta})\left|, \quad \varphi_{\alpha}(x, t)=D_{\alpha} \Phi(x, t, \theta, \bar{\theta})\right|, \\
F(x, t) & =D^{2} \Phi(x, t, \theta, \bar{\theta}) \mid .
\end{aligned}
$$

The equivalent fields for the $B$ superfield are simply marked by a subscripted $B$. The detailed structure of eq. (6) in components fields is:

$$
\begin{aligned}
\left(\begin{array}{c}
\partial_{t} A \\
\partial_{t} \bar{F}
\end{array}\right) & =\left(\begin{array}{cc}
M & 1 \\
\square & M
\end{array}\right)\left(\begin{array}{c}
\bar{F}+V^{\prime}(A)+2 A_{B} \\
\square A+V^{\prime \prime}(\bar{A}) \bar{F}+g \bar{\varphi} \bar{\varphi}+2 \bar{F}_{B}
\end{array}\right), \\
\left(\begin{array}{c}
\partial_{t} \varphi_{\alpha} \\
\partial_{t} \bar{\varphi}^{\dot{\alpha}}
\end{array}\right) & =\left(\begin{array}{cc}
M & \partial_{\alpha \dot{\alpha}} \\
\partial^{\dot{\alpha} \alpha} & M
\end{array}\right)\left(\begin{array}{c}
(\not \partial \bar{\varphi})_{\alpha}+V^{\prime}(A) \varphi_{\alpha}+2\left(\varphi_{B}\right)_{\alpha} \\
(\not \partial \varphi)^{\dot{\alpha}}+V^{\prime}(\bar{A}) \bar{\varphi}^{\dot{\alpha}}+2\left(\varphi_{B}\right)^{\dot{\alpha}}
\end{array}\right) .
\end{aligned}
$$

Eq. (8) must be completed by its complex conjugate to give the evolution of $\bar{A}$ and $F$. This set of equations is by construction invariant under supersymmetry. Notice that it turns the field $F$, which is just an auxiliary field in the usual approach, into a propagating field. Therefore, if one keeps the auxiliary field to maintain supersymmetry in a linear realization, the bulk formulation implies its propagation.

In the heuristic language of stochastic quantization, the $t$ evolution of $A, F$ and $\varphi$ is driven by the four-dimensional equations of motion and $F_{B}, A_{B}$ and $\varphi_{B}$, their respective noises. The form of the kernels that multiply the combination of equations of motion and noises is dictated by the requirement that the $t$ evolution is compatible with supersymmetry and the respective dimensions of the fields and their equations of motion. We believe that eqs. (8.9) open a way toward a non-perturbative definition of supersymmetric theories.

The ghost completion of eq. (5) is very easy to derive from eq. (†). It is such that the full action is BRS-invariant. For correlators at equal time $t$ of fields $\Phi$ and $B$ only, which are sufficient to determine the $S$-matrix of the model, the ghosts can be integrated out without destroying supersymmetry. It is useful to write the ghost equations of motion:

$$
\begin{aligned}
\left(\begin{array}{c}
\partial_{t} \Psi \\
\partial_{t} \bar{\Psi}
\end{array}\right) & =\left(\begin{array}{cc}
M & \bar{D}^{2} \\
D^{2} & M
\end{array}\right)\left(\begin{array}{l}
\bar{D}^{2} \bar{\Psi}+V^{\prime \prime}(\Phi) \Psi \\
D^{2} \Psi+V^{\prime \prime}(\bar{\Phi}) \bar{\Psi}
\end{array}\right), \\
\left(\begin{array}{l}
-\partial_{t} \chi \\
-\partial_{t} \bar{\chi}
\end{array}\right) & =\left(\begin{array}{cc}
M & \bar{D}^{2} \\
D^{2} & M
\end{array}\right)\left(\begin{array}{l}
\bar{D}^{2} \bar{\chi}+V^{\prime \prime}(\Phi) \chi \\
D^{2} \chi+V^{\prime \prime}(\bar{\Phi}) \bar{\chi}
\end{array}\right) .
\end{aligned}
$$

We must now precise the symmetry with respect to the reversal of the bulk time $t$, which implies that the $s$-invariant action is really of the form (4). Moreover this symmetry implies the stability of the theory under radiative corrections. It reads as follows:

$$
\begin{aligned}
t & \rightarrow-t, \quad \Phi(x, t) \rightarrow \Phi(x,-t), \\
\Psi(x, t) & \rightarrow \chi(x,-t), \quad \chi(x, t) \rightarrow-\Psi(x,-t), \\
B(x, t) & \rightarrow-H(x,-t) .
\end{aligned}
$$


This discrete symmetry explicitly depends on the dynamics, due to the appearance of the classical equations of motion in $H$. Notice that the renormalization of vertices with only external lines of $\Phi$ and $B$ fields does not involve the ghosts, since the latter have retarded propagators and do not contribute to closed loops. This symmetry completely justifies the expression ( $₫$ ) of the action. We also see that the equations of motion (6) are invariant under the symmetry (12).

\section{Supersymmetric Gauge Theories}

For theories with a gauge symmetry, quantization with a ghost time involves an additional subtlety. The topological symmetry in five dimension must be disentangled from the gauge symmetry. This problem has been recently solved by introducing a second BRST symmetry $w$ which anticommutes with $s$. The theory is then defined as being invariant under both $s$ and $w$. Due to the topological character of the $s$-symmetry, the cohomology of $s$ is empty. The observables are defined from the cohomology of $w$. The full complement of fields necessary to realize both symmetries is described in [ [ for the Yang-Mills theory.

This general construction should work also in the supersymmetric case. Technical difficulties however arise and the subject deserves a separate publication [6]. In the following, we restrict ourselves to present the basic features in the simpler SQED case and sketch the basic problems.

The supersymmetric Maxwell field is described by a real superfield $V$ of dimension 0 , with a chiral gauge parameter $\Lambda$ acting by $V \rightarrow V+i(\Lambda-\bar{\Lambda})$. The gauge invariant field strength is the chiral superfield $W_{\alpha}=\bar{D}^{2} D_{\alpha} V$ and its complex conjugate $\bar{W}_{\dot{\alpha}}$. The equation of motion is $D^{\alpha} W_{\alpha}=0$, which is equivalent to $D^{\dot{\alpha}} \bar{W}_{\dot{\alpha}}=0$ by the Bianchi identity and is of dimension 2. But we cannot simply combine the study of gauge theories of [ $₫$ ] with a superspace formalism. In superspace, the gauge parameter becomes a full multiplet, which gives algebraic gauge variations to some components of the superfield $V$. It is therefore necessary to verify that all the components of the superfield $V$, either physical fields or pure gauges, have well-defined propagators.

The topological BRST symmetry is similar to the one for the Wess-Zumino model, but with additional terms that account for the gauge symmetry in superspace. A chiral superfield $X$ must be introduced. It is the supersymmetric generalization of the gauge field component $A_{5}$ that plays a key role for the bulk quantization of the genuine Yang-Mills theory [4, 5]. We introduce chiral superfields $C$ and $\phi$ as additional ghost companions to $X, \Psi_{X}$ :

$$
\begin{aligned}
s V & =\Psi_{V}+i(C-\bar{C}), \quad s \Psi_{V}=-i(\phi-\bar{\phi}), \\
s C & =\phi, \quad s \phi=0, \\
s X & =\Psi_{X}+\partial_{t} C, \quad s \Psi_{X}=-\partial_{t} \phi .
\end{aligned}
$$

We have the antighosts $\Psi_{V}^{b}$ and $\phi^{b}$ and the corresponding Lagrange multipliers $B_{V}$ and $\eta$ :

$$
s \Psi_{V}^{b}=B_{V}, \quad s B_{V}=0, \quad s \phi^{b}=\eta, \quad s \eta=0 .
$$

We suppose that the full $w$ and $s$ BRS-like symmetries will require additional fields, as they had to be introduced in the Yang-Mills case [4, 5], but we do not go in these details now. 
The Lagrangian will be written with the combination of the equations of motion and noise $B_{V}+D^{\alpha} \bar{D}^{2} D_{\alpha} V$. We expect to obtain the following $t$-evolution of the superfield $V$ :

$$
\partial_{t} V=B_{V}+D^{\alpha} \bar{D}^{2} D_{\alpha} V+i(X-\bar{X}) .
$$

The last term is necessary to determines the $t$ evolution over the supersymmetric generalization of gauge orbits and justifies the introduction of the superfield $X$.

Now comes the difficulty. The later equation must be derived from a Lagrangian that is built from the $s$-variation of the integral in superspace of $\Psi_{V}^{b}$ times the desired variation of $V$ given in eq. (15),

$$
\int d t d^{4} x d^{4} \theta s\left(\Psi_{V}^{b}\left(\partial_{t} V-D^{\alpha} \bar{D}^{2} D_{\alpha} V+B_{V}-i(X-\bar{X})\right)\right) .
$$

Variation with respect to an unconstrained $B_{V}$ yields equation (15), but for them to have the correct physical interpretation, we need the linear constraint on $B_{V}, D^{2} B_{V}=$ 0 and $\bar{D}^{2} B_{V}=0$. Without such a constraint, the auxiliary $D$-field would eventually appear as a propagating scalar, since its equation of motion from eq. (15) is of the type $\partial_{t} D=\square D+B_{D}$, where $B_{D}$ is the scalar auxiliary component in $B_{V}$. In the constrained linear multiplet, this higher component of the field is equal to the Laplacian acting on the scalar field of the multiplet. Similarly, the equation of motion of the photino would be $\partial_{t} \lambda=\square \lambda+B_{\lambda}$. The constraint on $B_{V}$ insures that $B_{\lambda}$ is equal to the Dirac operator acting on the fermionic component of the supermultiplet, which yields the correct interpretation. However, variation with respect to a constrained field would not produce eq. (15), but some superspace derivative of it. In this case, the equation would act only on the field strength $W_{\alpha}$, letting the gauge variant part of the field $V$ without a definite evolution.

These difficulties seem to be related to the fact that the detailed balance of degrees of freedom between fields of different types is not so simple to obtain as in the WessZumino model. The effective number of degrees of freedom of the gauge field $V$ is reduced by the gauge symmetry, but this mechanism is not available for the antighost and noise. In fact, these are quite similar to the canonical momenta in Hamiltonian gauge field theory, which are constrained by Gauß' law. The equivalent superfield constraint is satisfied by the field equations and defines a linear superfield. (Let us recall that a linear superfield $l$ is a real superfield satisfying $D^{2} l=0$ and $\bar{D}^{2} l=0$; its independent components are a scalar, a Majorana spinor and a divergenceless vector field.) As in the quantization of the Hamiltonian gauge field theory, one would wish to express this constraint as the equation obtained from the variation of the time component of the connection. In superspace, the connection is not an ordinary vector and what play the role of the time-like component of the connection is a chiral superfield, which transforms as the time derivative of the chiral superfield gauge parameter.

\section{Conclusion}

In this letter, we have shown that the quantization with an additional time can be defined while maintaining supersymmetry on the example of the Wess-Zumino model. Since this method of quantization is expected to be more profound from a non perturbative point of view, this is a progress in view of further tests for supersymmetry. In 
a separate publication, we will explain how our result can be extended to the case of supersymmetric gauge theories.

Acknowledgements: We thank Luis Alvarez-Gaumé for very useful discussions concerning this work. The research of Laurent Baulieu was supported in part by DOE grant DE-FG02-96ER40959.

\section{References}

[1] G. Parisi and Y.S. Wu. Sci. Sinica 24 (1981), p. 484.

[2] P.H. Daamgard and H. Huffel. Phys. Rep. 152 (1983), p. 227.

[3] Laurent Baulieu and Daniel Zwanziger, $Q C D_{4}$ From a Five-Dimensional Point of View. Nucl. Phys. B 581 (2000), pp. 604-640. hep-th/9909006.

[4] Laurent Baulieu, Pietro Antonio Grassi, and Daniel Zwanziger. Gauge and Topological Symmetries in the Bulk Quantization of Gauge Theories. hep-th/0006036, to appear in Nucl. Phys. B.

[5] Laurent Baulieu and Daniel Zwanziger. From stochastic quantization to bulk quantization: Schwinger-Dyson equations and S-matrix. hep-th/0012103.

[6] Laurent Baulieu and Marc Bellon. Bulk quantization of supersymmetric gauge theories. in preparation, (2001). 